\title{
Tendência de declínio da taxa de mortalidade por suicídio no Paraná, Brasil: contribuição para políticas públicas de saúde mental
}

\author{
Trend of declining suicide mortality rate in Paraná, Brazil: \\ contribution to public mental health policies \\ Natalina Maria da Rosa', Cátia Millene Dell Agnolo', Rosana Rosseto de Oliveira', Thais Aidar de Freitas Mathias ${ }^{1}$
}

\section{RESUMO}

\section{Palavras-chave}

Suicídio, tendência, saúde mental.

\section{Keywords}

Suicide, trend, mental health.
Objetivo: Analisar a tendência da mortalidade por suicídio no Estado do Paraná, Brasil. Métodos: Estudo ecológico, de séries temporais, com análise da tendência da mortalidade por suicídio no Estado do Paraná, Brasil, utilizando dados do Sistema de Informações sobre Mortalidade de 1996 a 2012, por meio de regressão polinomial. Resultados: Houve tendência decrescente de 0,16 ao ano na taxa de mortalidade por suicídio no Estado do Paraná e aumento da taxa de mortalidade apenas para a Regional de Saúde de Paranaguá (0,19 ao ano). Verificou-se que o sexo masculino apresentou maior taxa de mortalidade por suicídio, com queda significativa para os homens com idade de 65 anos ou mais. Conclusões: A maioria das Regionais de Saúde do Estado do Paraná apresentou tendência decrescente nas taxas de mortalidade por suicídio. Contudo, houve variações da tendência entre as regiões, faixas etárias e sexo, com maior queda na taxa de mortalidade por suicídio no sexo masculino, com idade de 65 anos ou mais. A análise de tendência da mortalidade por suicídio reportou ser um importante instrumento para identificar as regiões no Paraná que necessitam de ações diretivas de promoção à saúde mental.

\section{ABSTRACT}

Objective: To analyze the trend of suicide mortality in Paraná State, Brazil. Methods: Ecological, time series study, with analysis of trend of suicide mortality in Paraná State, Brazil, using data of the Mortality Information System from 1996 to 2012, by polynomial regression. Results: There was a decreasing trend of 0.16 per year in the suicide mortality rates in the Paraná State and an increased mortality rate only for the Healthcare Region of Paranaguá. It was verified that the male sex had a higher death rate due to suicide, with a significant decrease for men aged 65 years and over. Conclusions: The majority of the Health Region of the State of Paraná presented a decreasing trend in mortality rates due to suicide. However, there were variations in the trend between regions, age groups and sex, with the highest drop in mortality rate due to suicide in males aged 65 years and over. The trend analysis of suicide mortality has been an important tool to identify the regions in Paraná that require directives actions to promote mental health.

1 Universidade Estadual de Maringá (UEM), Programa de Pós-Graduação em Enfermagem.
Recebido em

$21 / 10 / 2016$

Aprovado em

$22 / 07 / 2017$ 


\section{INTRODUÇÃO}

O suicídio representa uma das 20 principais causas de morte no mundo, se consideradas todas as faixas etárias'. Caracteriza-se como um grave problema de saúde pública, pelo seu aumento progressivo em nível mundial. Estima-se que 900 mil pessoas cometem suicídio ao ano, o que caracteriza a ocorrência de um ato suicida a cada 40 segundos e uma tentativa de suicídio a cada três segundos. De acordo com a Organização Mundial da Saúde (OMS), em 2020 o número de óbitos por suicídio alcançará cerca de 1.500 mil casos².

Relatório mundial divulgado em setembro de 2014 pela OMS inferiu que o número absoluto de óbitos por suicídio pode ser superior aos dados disponibilizados nos sistemas mundiais de informação sobre mortalidade. Comumente, a maior parte dos óbitos por suicídio ou tentativas de suicídio não chega aos registros oficiais porque não existe notificação compulsória para esse fenômeno como ocorre para muitos outros agravos ${ }^{3}$.

Mesmo com a subnotificação de grande parte dos registros de suicídio, as taxas globais de mortalidade por suicídio tiveram acréscimo de $60 \%$ nos últimos 45 anos $^{3}$, e alguns países se destacaram por apresentar aumento percentual elevado na taxa de mortalidade por suicídio, como Mauritius entre o período de 1981 a 2011 (aumento de 368,4\%, passando de 1,9 em 1981 para 8,9 óbitos por 100 mil habitantes em 2011); República da Coreia, entre 1985 e 2011 (161,6\%, 9,9-25,9 óbitos por 100 mil habitantes); e Trinidad e Tobago, de 1979 a 2008 (149,1\%, 5,7-14,6 óbitos por 100 mil habitantes)4.

Nos países da América do Sul, entre 1996 e 2012, as taxas de mortalidade por suicídio apresentaram aumento percentual progressivo e constante. No Uruguai o aumento foi de 40,4\% (11,4 para 16,0 óbitos por 100 mil habitantes); na Argentina, 16,9\% (6,5 para 7,6 óbitos por 100 mil habitantes); no Equador, 16,4\% (5,5 para 6,4 óbitos por 100 mil habitantes); no Paraguai, as taxas duplicaram, passando de 2,4 para 4,4 óbitos por 100 mil habitantes, aumento acentuado de 83,3\%4; e, em especial, no Brasil, a taxa de mortalidade por suicídio teve crescimento de 23,3\% (4,3 para 5,3 óbitos por 100 mil habitantes) $)^{5}$.

Por ser um problema complexo e multifatorial, a abordagem do tema suicídio pelo sistema público de saúde é problemática, sendo necessários a sensibilização e o envolvimento de profissionais de diferentes setores ${ }^{6}$. Pesquisas sobre a mortalidade por suicídio no Estado do Paraná ainda são escassas, o que sinaliza a necessidade de conhecer e compreender o comportamento das taxas de mortalidade por suicídio, a fim de possibilitar a determinação do grau de influência dos contextos socioeconômico, cultural, político e psicossocial sobre esse evento e contribuir para a prevenção de sua ocorrência.

Dessa forma, este estudo teve por objetivo analisar a tendência da taxa de mortalidade por suicídio no Estado do
Paraná, Brasil, entre os anos de 1996 a 2012, segundo sexo, idade, Macrorregionais e Regionais de Saúde.

\section{MÉTODOS}

Trata-se de um estudo descritivo, ecológico, do tipo série temporal, sobre a mortalidade por suicídio no Estado do Paraná, Brasil, de 1996 a 2012. A população residente nos 399 municípios do Paraná está disposta em 22 Regionais de Saúde (RS), que, por sua vez, estão agrupadas em quatro Macrorregionais de Saúde: Leste, Norte, Noroeste e Oeste.

Para a análise da mortalidade por suicídio foram utilizados dados do Sistema de Informações sobre Mortalidade (SIM) e dados da população residente no Paraná do Instituto Brasileiro de Geografia e Estatística (IBGE). Esses dados são de domínio público e ambos estão disponíveis no Departamento de Informática do Sistema Único de Saúde (Datasus). Foram selecionados os óbitos de residentes no Paraná, com 15 anos ou mais de idade, entre o período de 1996 a 2012, do agrupamento de lesões autoprovocadas intencionalmente (X60-X84), codificados segundo a décima revisão da Classificação Estatística Internacional de Doenças e Problemas Relacionados à Saúde (CID-10) ${ }^{7}$. A idade foi estratificada segundo critério da $\mathrm{OMS}^{7}, 15$ a 44, 45 a 64, e 65 anos e mais. As taxas de mortalidade por 100 mil habitantes foram calculadas para cada Macrorregional e Regional de Saúde, por sexo e idade.

A análise de tendência foi realizada por meio do modelo de regressão polinomial. Para a modelagem, a taxa de mortalidade foi considerada variável dependente (y) e os anos de período de estudo, variável independente $(\mathrm{x})$. Realizou-se a centralização da variável "ano" pelo ponto médio da série histórica. Para suavizar a série em razão da grande oscilação dos pontos, calculou-se a média móvel centrada em três termos. O primeiro modelo testado foi o de regressão linear simples e, em seguida, os modelos de segundo e terceiro graus. Calculou-se o coeficiente de determinação $\left(r^{2}\right)$ como medida de magnitude da variância da taxa de mortalidade por suicídio, explicada pela variância da variável preditora ano. Considerou-se como modelo mais adequado aquele que apresentasse resíduo sem vícios, significância estatística $(p<0,05)$ e melhor medida de precisão $\left(r^{2}\right)^{8}$. Para as análises estatísticas, utilizou-se o software Statistical Package for the Social Sciences (SPSS) versão 21.0.

Para avaliar o comportamento das taxas de mortalidade por suicídio nas RS, foram classificadas como baixas as taxas que não atingissem cinco óbitos por 100 mil habitantes; médias as que se encontravam entre cinco e menos de 15 óbitos por 100 mil habitantes; elevadas as taxas entre 15 e menos de 30 óbitos por 100 mil habitantes; e muito elevadas as taxas com 30 ou mais óbitos por 100 mil habitantes 9 . Este estudo foi aprovado pelo Comitê de Ética em Pesquisa da Universidade Estadual de Maringá (protocolo no 781.253/2014). 


\section{RESULTADOS}

No Estado do Paraná, Brasil, entre 1996 e 2012, ocorreram 10.344 óbitos por suicídio, distribuídos entre as quatro Macrorregionais: Leste (4763), Norte (1866), Noroeste (1605) e Oeste (2110). A mortalidade por suicídio diminuiu, em média, 0,16 óbitos a cada 100 mil habitantes ao ano. Nesse período, constatou-se tendência decrescente nas taxas de mortalidade por suicídio, com declínio da taxa em 15 RS, tendência constante em sete RS e aumento na taxa de mortalidade por suicídio apenas para a RS de Paranaguá. No entanto, dentre as Macrorregionais de Saúde, a Oeste foi a que atingiu maior taxa média de mortalidade por suicídio em todos os períodos (Tabela 1).

Ao ser analisado por sexo, verificou-se que a taxa média entre os homens foi superior à das mulheres. Entretanto, mesmo com menores taxas, o sexo feminino apresentou maior queda na mortalidade por suicídio quando considerado o Estado em sua totalidade (Figura 1).

Na Figura 2 observa-se o declínio das taxas de mortalidade por suicídio em todas as faixas etárias para homens e mulheres. No entanto, destaca-se que o sexo masculino com idade de 65 anos ou mais e a população feminina com idade de 15 a 44 anos tiveram taxa média mais elevada.

Nos anos de 1996 a 2000, houve maior concentração de taxas de mortalidade por suicídio consideradas médias e elevadas para ambos os sexos. A população do sexo masculino teve o maior número de RS com taxas elevadas de mortalidade por suicídio. E a população do sexo feminino, por sua vez, atingiu taxas de mortalidade por suicídio classificadas como médias. Já no período de 2009 a 2012, houve redução de RS com taxas elevadas de mortalidade por suicídio para o sexo masculino e médias para o feminino (Figura 3).

Tabela 1. Tendência das taxas de mortalidade por suicídio, segundo Macrorregional e Regional de Saúde, Paraná, Brasil, 1996 a 2012.

\begin{tabular}{|c|c|c|c|c|c|c|c|c|}
\hline \multirow{2}{*}{$\begin{array}{l}\text { Macrorregional/ } \\
\text { Regional de Saúde }\end{array}$} & \multirow{2}{*}{$\begin{array}{c}1996 / 2000 \\
\text { Taxa } \\
\end{array}$} & \multirow{2}{*}{$\begin{array}{c}2001 / 2004 \\
\text { Taxa } \\
\end{array}$} & \multirow{2}{*}{$\begin{array}{c}2005 / 2008 \\
\text { Таха } \\
\end{array}$} & \multirow{2}{*}{$\frac{2009 / 2012}{\text { Taxa }}$} & \multirow{2}{*}{$\beta 1$} & \multirow{2}{*}{$\mathbf{r}^{2}$} & \multirow{2}{*}{$\mathrm{p}$} & \multirow{2}{*}{ Tendência* } \\
\hline & & & & & & & & \\
\hline Leste & 8,5 & 8,1 & 7,9 & 6,7 & 0,14 & 0,68 & $<0,001$ & $\downarrow$ \\
\hline 1a - Paranaguá & 5,6 & 6,7 & 8,8 & 7,5 & $+0,19$ & 0,45 & 0,006 & $\uparrow$ \\
\hline $2^{\mathrm{a}}-$ Metropolitana & 7,1 & 6,8 & 6,3 & 5,1 & $-0,17$ & 0,63 & $<0,001$ & $\downarrow$ \\
\hline $3^{a}$ - Ponta Grossa & 10,3 & 8,9 & 8,3 & 7,8 & $-0,13$ & 0,52 & 0,002 & $\downarrow$ \\
\hline $4^{\mathrm{a}}-$ Irati & 11,2 & 10,9 & 11,4 & 8,7 & $-0,25$ & 0,54 & 0,002 & $\downarrow$ \\
\hline $5^{\mathrm{a}}-$ Guarapuava & 13,4 & 12,2 & 12,1 & 10,6 & $-0,20$ & 0,54 & 0,002 & $\downarrow$ \\
\hline 6a - União da Vitória & 10,5 & 13,3 & 10,6 & 13,1 & $+0,60$ & 0,05 & 0,383 & - \\
\hline $7^{\mathrm{a}}-$ Pato Branco & 13,9 & 10,9 & 15,7 & 9,6 & $-0,14$ & 0,10 & 0,25 & - \\
\hline 21a - Telêmaco Borba & 7,0 & 10,4 & 10,0 & 9,4 & $+0,14$ & 0,14 & 0,163 & - \\
\hline Norte & 9,8 & 8,3 & 7,0 & 7,8 & $-0,19$ & 0,9 & $<0,001$ & $\downarrow$ \\
\hline $16^{\mathrm{a}}$ - Apucarana & 10,8 & 8,6 & 7,7 & 10,6 & $-0,07$ & 0,05 & 0,413 & - \\
\hline 17a - Londrina & 7,9 & 7,3 & 4,8 & 5,8 & $-0,21$ & 0,70 & $<0,001$ & $\downarrow$ \\
\hline 18 - Cornélio Procópio & 11,4 & 9,1 & 8,2 & 6,8 & $-0,37$ & 0,75 & $<0,001$ & $\downarrow$ \\
\hline 19a - Jacarezinho & 11,0 & 8,6 & 10,5 & 10,0 & $-0,49$ & 0,38 & 0,484 & - \\
\hline $22^{\mathrm{a}}$ - Ivaiporã & 13,2 & 12,0 & 10,1 & 10,9 & $-0,20$ & 0,51 & 0,006 & $\downarrow$ \\
\hline Noroeste & 8,8 & 7,8 & 7,2 & 7,0 & $-0,17$ & 0,77 & $<0,001$ & $\downarrow$ \\
\hline $11^{\mathrm{a}}$ - Campo Mourão & 11,8 & 11,3 & 11,4 & 8,3 & $-0,30$ & 0,45 & 0,006 & $\downarrow$ \\
\hline $12^{\mathrm{a}}$ - Umuarama & 10,5 & 9,1 & 8,4 & 7,0 & $-0,31$ & 0,73 & $<0,001$ & $\downarrow$ \\
\hline $13^{\mathrm{a}}$ - Cianorte & 7,3 & 11,1 & 6,6 & 6,0 & $-0,7$ & 0,63 & 0,003 & $\downarrow$ \\
\hline 14a - Paranavaí & 8,6 & 6,1 & 5,0 & 8,4 & $-0,09$ & 0,08 & 0,317 & - \\
\hline 15a - Maringá & 6,9 & 5,6 & 5,7 & 6,2 & $-0,06$ & 0,56 & 0,003 & $\downarrow$ \\
\hline Oeste & 11,7 & 12,6 & 10,5 & 10,1 & $-0,40$ & 0,84 & $<0,001$ & $\downarrow$ \\
\hline $8^{\mathrm{a}}-$ Francisco Beltrão & 15,1 & 16,4 & 12,2 & 15,1 & $-0,17$ & 0,28 & 0,041 & $\downarrow$ \\
\hline 9a - Foz do Iguaçu & 6,9 & 11,0 & 7,6 & 7,8 & - & - & 0,956 & - \\
\hline 10a - Cascavel & 11,4 & 11,4 & 9,9 & 8,2 & $-0,60$ & 0,77 & $<0,001$ & $\downarrow$ \\
\hline $20^{\mathrm{a}}-$ Toledo & 14,2 & 12,4 & 13,5 & 10,9 & $-0,24$ & 0,43 & 0,008 & $\downarrow$ \\
\hline Paraná & 9,3 & 8,8 & 8,0 & 7,5 & $-0,16$ & 0,92 & $<0,001$ & $\downarrow$ \\
\hline
\end{tabular}

* $\uparrow$ Crescente; $\downarrow$ Decrescente; - Constante. 

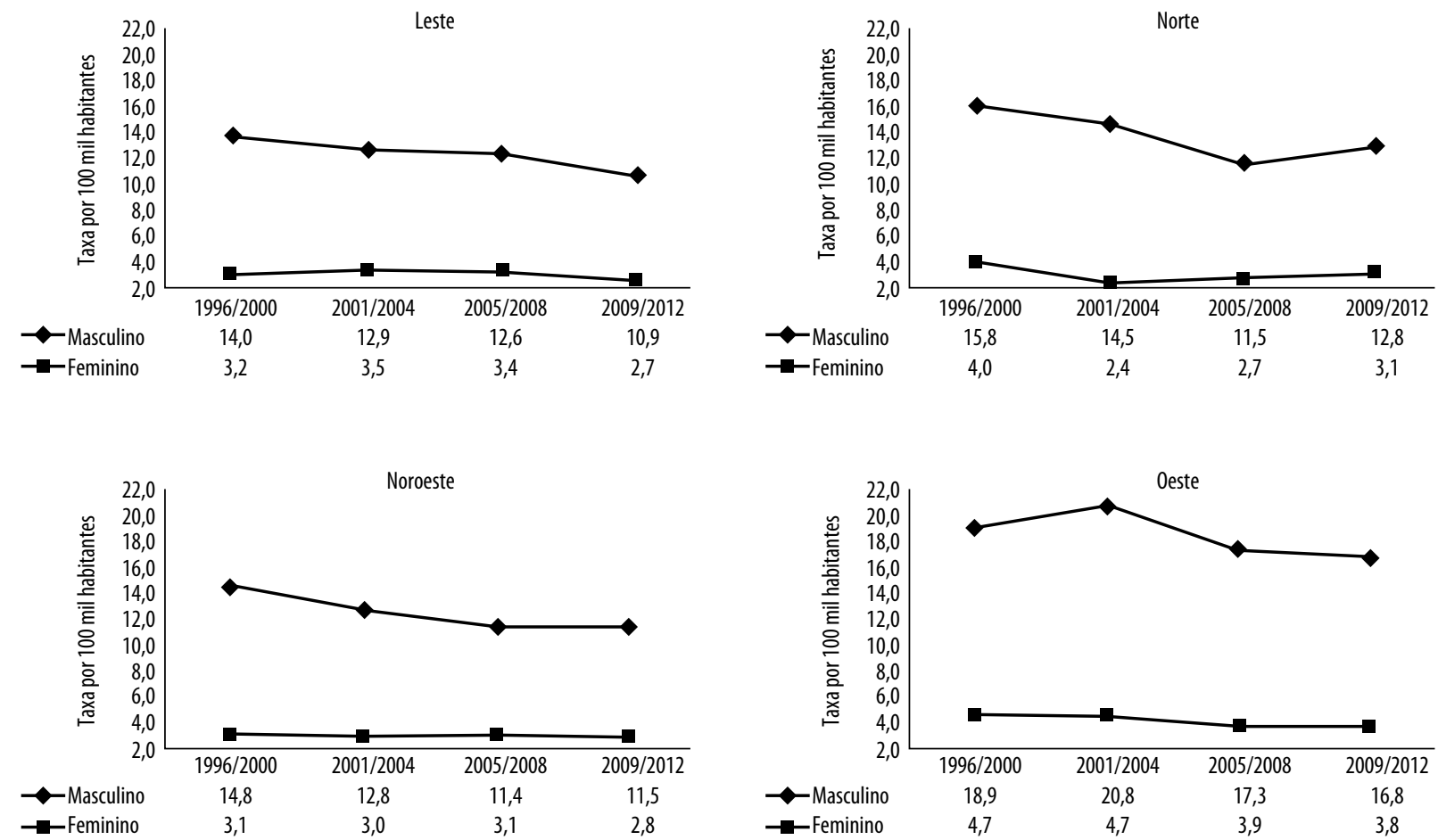

\begin{tabular}{|lcccc|}
\hline Macrorregional & $\boldsymbol{\beta 1}$ & $\mathbf{r}^{2}$ & $\mathbf{p}$ & Tendência \\
\hline Masculino & & & & \\
Leste & $-0,25$ & 0,77 & $<0,001$ & Decrescente \\
Norte & $-0,32$ & 0,68 & $<0,001$ & Decrescente \\
Noroeste & $-0,32$ & 0,76 & $<0,001$ & Decrescente \\
Oeste & $-0,61$ & 0,80 & $<0,001$ & Decrescente \\
Paraná & $-0,28$ & 0,95 & 0,001 & Decrescente \\
Feminino & & & & \\
Leste & $-0,03$ & 0,19 & 0,106 & Constante \\
Norte & $-0,06$ & 0,79 & $<0,001$ & Decrescente \\
Noroeste & 0,29 & 0,036 & Decrescente \\
Oeste & $-0,40$ & 0,26 & 0,052 & Constante \\
Paraná & $-0,05$ & 0,57 & 0,001 & Decrescente \\
\hline
\end{tabular}

Figura 1. Taxa de mortalidade por suicídio e respectivos modelos de tendência, segundo sexo e Macrorregional de Saúde. Paraná, Brasil, 1996 a 2012.

\section{DISCUSSÃO}

De forma geral, o Estado do Paraná, as Macrorregionais de Saúde e a maioria das RS apresentaram tendência decrescente nas taxas de mortalidade por suicídio, com variações entre as RS, sexo e faixa etária.

Embora o Paraná não tenha implementado estratégias voltadas para a prevenção e controle da mortalidade por suicídio, o Estado vem reestruturando a rede de atendimento à saúde mental com base na lógica territorial, ao longo dos anos. Com a instauração da Política Estadual de Saúde Mental (2001), a implementação e o planejamento de ações nessa área passaram a ser de responsabilidade municipal. Mediante a nova política, o Estado passou a investir maiores recursos financeiros em ambulatórios para atendimento em saúde mental, com maior controle na dispensação de medicamentos e supervisão da população sob risco, além de leitos em emergência psiquiátrica em hospitais gerais, Centros de Atenção Psicossocial (CAPS) e na Atenção Primária à Saúde (APS) ${ }^{10}$.

Partindo desse pressuposto, a instauração de medidas na APS pode intensificar o controle da mortalidade por suicídio na comunidade local. Somado a essa medida, os profissionais das unidades de urgência e emergência intra-hospitalar 

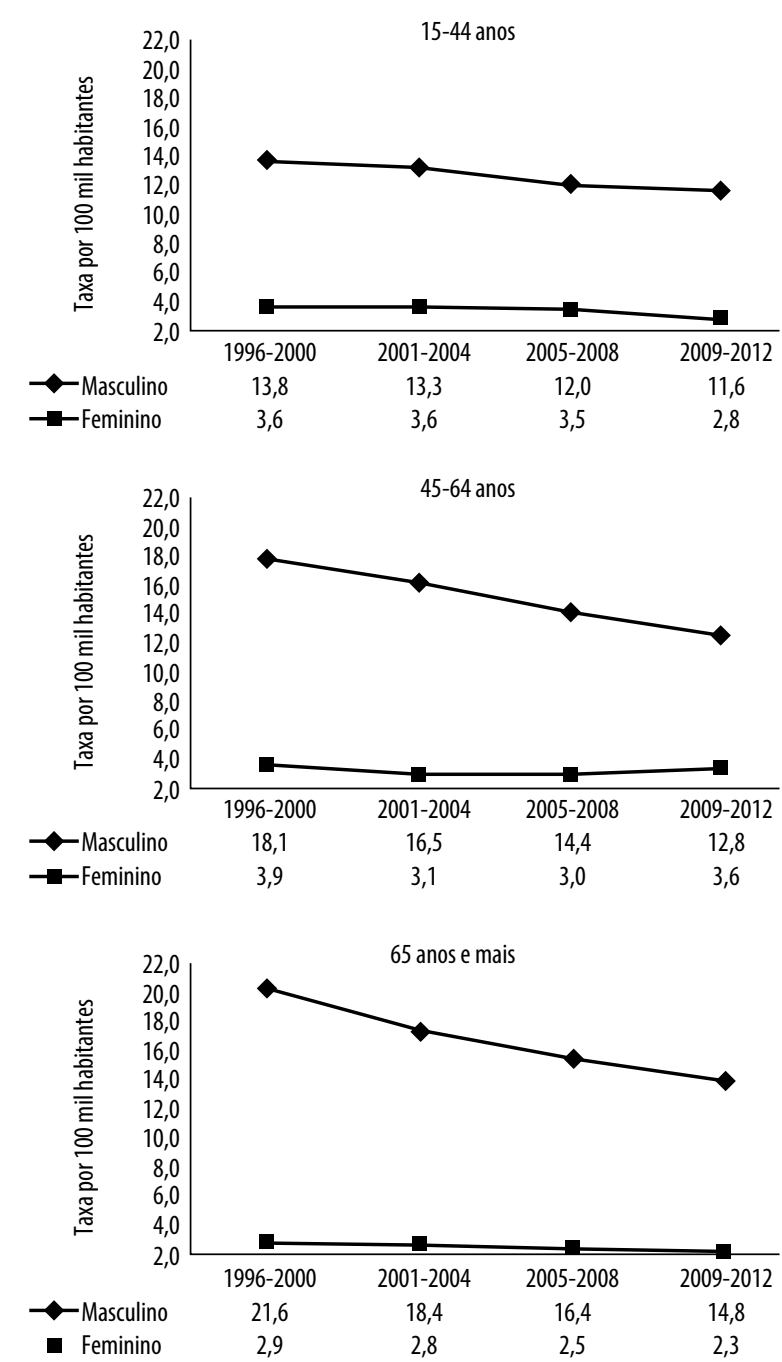

\begin{tabular}{|lccccc|}
\hline Idade & $\boldsymbol{\beta}$ & $\boldsymbol{\beta}_{1}$ & $\mathbf{r 2}$ & $\mathbf{p}$ & Tendência \\
\hline Masculino & & & & & \\
15 a 44 anos & 12,7 & $-0,18$ & 0,86 & $<0,001$ & Decrescente \\
45 a 64 anos & 15,7 & $-0,52$ & 0,96 & $<0,001$ & Decrescente \\
65 anos e mais & 17,4 & $-0,62$ & 0,82 & $<0,001$ & Decrescente \\
Feminino & & & & & \\
15 a 44 anos & 3,3 & $-0,05$ & 0,32 & 0,029 & Decrescente \\
45 a 64 anos & 3,0 & $-0,05$ & 0,56 & 0,003 & Decrescente \\
65 anos e mais & 2,7 & $-0,05$ & 0,33 & 0,026 & Decrescente \\
\hline
\end{tabular}

Figura 2. Taxa de mortalidade por suicídio e respectivos modelos de tendência, segundo idade e sexo. Paraná, Brasil, 1996 a 2012.

também podem contribuir para a redução da mortalidade por suicídio, contrarreferenciando potenciais suicidas para acompanhamento e tratamento na APS por toda a equipe de saúde, ou, em casos de transtornos mentais mais graves, encaminhando-os aos CAPS 6,10 .

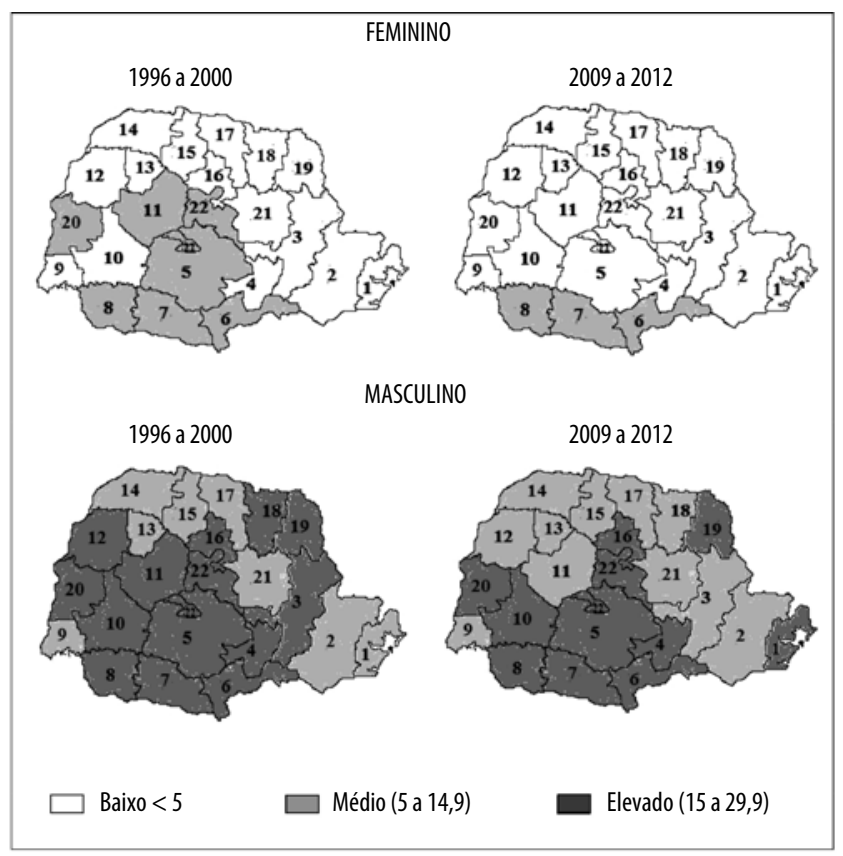

Figura 3. Distribuição das taxas de mortalidade por suicídio (por 100 mil habitantes) por Regionais de Saúde, segundo sexo. Paraná, Brasil, 1996 a 2000 e 2009 a 2012.

Vale ressaltar que, embora os paranaenses portadores de transtornos mentais possam ser assistidos pelos 2.662 estabelecimentos da APS e por aproximadamente 142 unidades de CAPS, a Macrorregional Oeste não dispõe de um número suficiente de CAPS para atender toda população, o que pode ser um dos fatores responsáveis pela elevada taxa de mortalidade por suicídio nessa macrorregional. Diante disso, faz-se necessário maior envolvimento dos profissionais da APS na identificação e assistência às pessoas com transtorno mental leve ou moderado ${ }^{10}$.

O governo do Estado do Paraná, preocupado com a alta taxa de mortalidade das vítimas de trauma e as que apresentam problemas clínicos com risco iminente de vida, como a tentativa de suicídio, implantou o Sistema Integrado de Atendimento ao Trauma e

Emergência (SIATE) em meados de 1990 e, aproximadamente uma década depois, o Serviço de Atendimento Móvel de Urgência (SAMU), promovendo o declínio dos índices de mortalidade e de possíveis sequelas decorrentes de eventos por causas externas ${ }^{11}$. A habilidade na abordagem do potencial suicida associada à efetividade no tratamento pode reduzir o número de vidas perdidas por esse evento².

O declínio da taxa de mortalidade por suicídio também pode estar relacionado à assistência fornecida pelos quatro Centros de Informação e Assistência Toxicológica (CIAT) existentes no Estado. O fornecimento de informação e orientação sobre o diagnóstico, prognóstico, tratamento e prevenção das intoxicações e envenenamentos contribui para minimizar os agravos à saúde dos usuários do Sistema de Saúde em todo o território nacional12. 
Conforme verificado neste estudo, a tendência da taxa de mortalidade por suicídio não obedeceu a um padrão uniforme para todas as RS. Isso também foi demonstrado em uma análise mais ampliada realizada com todas as 27 Unidades Federadas (UF) do Brasil|5. No país, com exceção dos Estados do Paraná, Ceará, Goiás, Rio de Janeiro e Amapá, as outras 22 UF apresentaram crescimento na taxa de mortalidade por suicídio entre 2002 e 2012, com alguns Estados apresentando altas taxas de mortalidade por suicídio no final do período, tais como o Rio Grande do Sul $(10,9)$, Santa Catarina $(8,6)$, Mato Grosso do Sul $(8,4)$, Roraima $(8,1)$, o que pode explicar a elevada taxa nacional de mortalidade por suicídio ${ }^{5}$.

No presente estudo, a 1a RS - Paranaguá foi a única RS que apresentou tendência crescente na taxa de mortalidade por suicídio. O aumento gradativo médio na taxa de mortalidade por suicídio do sexo masculino pode ter contribuído para essa tendência. Dos sete municípios que compõem a $1^{\text {a }}$ RS, Paranaguá detém maior representatividade populacional (53,9\%), bem como $51,6 \%$ de todos os registros de óbitos por suicídio ${ }^{13}$. No campo da saúde mental, em 2012, o município teve aumento de $55 \%$ no número de dependentes químicos, e cerca de $40 \%$ da população residente apresentava algum tipo de transtorno mental, destacando-se a depressão ${ }^{14}$.

No Irã, a depressão e o uso de drogas de abuso foram responsáveis pelo aumento da mortalidade por suicídio em 75\%, entre 1990 a 2010, evidenciando a necessidade de meIhoria nas políticas públicas de saúde mental e atenção primária naquele país ${ }^{15}$.

Em nível mundial, em distintas nações, ações e estratégias têm sido reconhecidas pelo sucesso na redução das taxas de suicídio, principalmente as realizadas na APS, com acompanhamento da população de risco compartilhado com a atenção secundária e demais órgãos públicos ${ }^{16}$.

A identificação precoce de indivíduos com maior vulnerabilidade para o suicídio é uma das ações mais efetivas em termos de prevenção do suicídio. Ademais, os fatores de risco para o suicídio compreendem: sexo masculino, conflitos familiares, abuso de álcool e outras drogas, grupos étnicos específicos, ausência de apoio social, abandono e características sociodemográficas desfavoráveis como desemprego e pobreza ${ }^{15}$.

História de tentativas de suicídio anteriores e de pessoas com transtornos mentais como depressão, transtornos bipolares e associação da depressão com uso de álcool e outras drogas de abuso configura o perfil para alto risco para o suicídio. Cerca de $85 \%$ dos potenciais suicidas sofrem de depressão maior e as tentativas de autoextermínio para quem sofre de depressão maior ou de transtorno afetivo bipolar (TAB) são 25 vezes maiores do que a da população em geral $^{17}$. Em consonância com essa afirmação, quase 100\% dos casos de suicídios estão relacionados a doenças mentais sem tratamento adequado ${ }^{2}$.

Concernente ao comportamento das taxas de mortalidade por suicídio no Estado do Paraná, o presente estudo cons- tatou taxas elevadas, especialmente para o sexo masculino. Ademais, ao considerar as taxas de mortalidade por suicídio da região Sul, um estudo sobre a análise da evolução da mortalidade nos Estados brasileiros evidenciou que a população masculina residente nos Estados do Rio Grande do Sul e Santa Catarina figurou taxas com valores alarmantes, cerca de 35,6 e 27,3 óbitos por 100 mil habitantes, respectivamente ${ }^{18}$. Ao considerar as altas taxas de mortalidade por suicídio para o sexo masculino nos três estados da região Sul, torna-se imperativo a implementação urgente de medidas preventivas para combater esse evento.

Globalmente, a escolha de métodos com maior grau de letalidade para concluir o suicídio é uma das explicações para as altas taxas de mortalidade por suicídio entre os homens, sobretudo em adultos mais velhos e idosos ${ }^{19}$.

Em virtude do grande acometimento de idosos, pode-se ressaltar que, para alcançar êxito na prevenção da mortalidade por suicídio nesse grupo etário, a equipe de saúde deve identificar os portadores de doenças depressivas, doenças físicas - pertinentes a velhice, idosos isolados socialmente, que perderam a função profissional e, principalmente, os que fizeram tentativas anteriores de suicídio. Para os homens, a desvantagem econômica, somada ao momento da aposentadoria, provoca sentimentos de ruptura relacionais e perda de status de poder fora e dentro da família ${ }^{20,21}$.

Este estudo apresenta algumas limitações que devem ser consideradas na interpretação dos resultados, tais como o uso de banco de dados secundários, em que há a possibilidade de variabilidade na completude e fidedignidade das informações dos registros sobre óbitos por suicídio no Sistema de Informações sobre Mortalidade, que pode caracterizar possível falha das RS no preenchimento da declaração de óbito ou subnotificação do suicídio por inadequação dos registros. É preciso considerar ainda que as taxas de mortalidade por suicídio variam de acordo com aspectos culturais, regionais, sociodemográficos e também com a maneira como essas mortes são registradas ${ }^{22}$, uma vez que a imprecisão dos dados de mortalidade dificulta a análise estatística desse evento ${ }^{23}$.

No entanto, a qualidade do preenchimento das declarações de óbito no Paraná vem sofrendo melhorias, o que, além de favorecer a alimentação de dados mais precisos sobre mortalidade no banco de dados do SIM, propicia a realização de análises mais apuradas sobre a mortalidade por suicídio no Estado, dado o bom grau de confiabilidade dos registros ${ }^{24}$.

\section{CONCLUSÕES}

O Estado do Paraná, as Macrorregionais de Saúde e grande parte das RS apresentaram tendência decrescente nas taxas de mortalidade por suicídio de 1996 a 2012. Entretanto, a tendência não obedeceu a um padrão uniforme para todas 
as regiões, assim como entre as faixas etárias, sendo maior a queda na taxa de mortalidade por suicídio no sexo masculino com idade de 65 anos ou mais.

Nesse sentido, a análise de regressão polinomial utilizada mostrou-se uma importante ferramenta para identificar as regiões que necessitam de ações diretivas de promoção à saúde mental, uma vez que o conhecimento dessas taxas em cada RS permite identificar onde há maior necessidade de ações de saúde pública. Investigações das eventuais razões associadas às taxas de mortalidade por suicídio merecem novos estudos epidemiológicos aprofundados.

Ao considerar que na atual conjuntura o Estado do Paraná não contempla programas de prevenção do suicídio, promulga-se a urgente necessidade de capacitar a equipe de profissionais de saúde, para a identificação precoce de indivíduos da comunidade na iminência do suicídio.

\section{CONTRIBUIÇÕES INDIVIDUAIS}

Natalina Maria da Rosa - Contribuiu significativamente da concepção do projeto, coleta, análise e interpretação dos dados; redação do artigo; revisão crítica relevante do conteúdo intelectual e aprovação da versão final a ser publicada.

Cátia Millene Dell Agnolo - Contribuiu significativamente na análise e interpretação dos dados; redação do artigo; revisão crítica relevante do conteúdo intelectual e aprovação da versão final a ser publicada.

Rosana Rosseto de Oliveira - Contribuiu significativamente na análise e interpretação dos dados; revisão crítica relevante do conteúdo intelectual e aprovação da versão final a ser publicada.

Magda Lúcia Félix de Oliveira - Contribuiu significativamente na concepção do projeto de pesquisa, orientação, correção, revisão crítica relevante do conteúdo intelectual e aprovação da versão final do manuscrito.

Thais Aidar de Freitas Mathias - Contribuiu significativamente na revisão crítica relevante do conteúdo intelectual e aprovação final da versão a ser publicada.

\section{CONFLITOS DE INTERESSE}

As autoras não possuem conflitos de interesse a serem declarados.

\section{REFERÊNCIAS}

1. World Health Organization. Preventing suicide: a global imperative. 2014. Disponível em: http://www.who.int/mental_health/suicide-prevention/exe_summary_english. pdf?ua =1. Acesso em: 5 jan. 2014
2. Bertolote JM, Mello-Santos C, Botega NJ. Detecting suicide risk at psychiatric emergency services. Rev Bras Psiquiatr. 2010;32:587-95.

3. World Health Organization. Prevention: addressing specific risk factors. 2006-2007. 2014a. Disponível em: http://www.who.int/nmh/donorinfo/msd_preventing_suicide_update. pdf?ua=1. Acesso em: 3 jan. 2014.

4. World Health Organization. Mortality database. 2014b. Disponível em: http://apps.who. int/healthinfo/statistics/mortality/whodpms. Acesso em: 3 jan. 2014

5. Waiselfisz JJ. Mapa da violência 2014: os jovens do Brasil. Braślila: Flacso; 2014.

6. Moura ATM, Almeida EC, Rodrigues PHA, Nogueira RC, Santos TEEH, organizador. Prevenção do suicídio no nível local: orientações para a formação de redes municipais de prevenção e controle do suicídio e para os profissionais que a integram. Porto Alegre: CORAG; 2011

7. World Health Organization. International statistical classification of diseases and related health problems: instruction manual. 10 ${ }^{\text {th }}$ rev. Geneva, 2010. v. 2. 2014. Disponivel em: http://www.who.int/classifications/icd//CD10Volume2_en_2010.pdf. Acesso em: 20 mar. 2014

8. Latorre MRDO, Cardoso MRA. Análise de séries temporais em epidemiologia: uma introdução sobre os aspectos metodológicos. Rev Bras Epidemiol. 2001;4:145-52.

9. Diekstra RF, Gulbinat W. The epidemiology of suicidal behaviour: a review of three continents. World Health Stat Ann. 1993:46:52-68.

10. Paraná. Secretaria de Estado da Saúde. A Rede de Atenção à Saúde Mental no Paraná e a competência da APS. 2014. Disponível em: http://www.saude.pr.gov.br/arquivos/File/ APSUS_-_Ed._Permanente/Oficia_8__Saude_Mental/Apresentacao_Rede_de_ Atencao_a_Saude_Mental_no_Parana_e_a_competencia_da_APS.pdf. Acesso em: 10 fev. 2014

11. Magalhães APN, Alves VM, Comassetto I, Lima PC, Faro ACM, Nardi AE. Atendimento a tentativas de suicídio por serviço de atenção pré-hospitalar. J Bras Psiquiatr. 2014;63:16-22.

12. Lira SVG, Silva JG, Abreu RNDC, Moreira DP, Vieira LJES, Frota MA. Intoxicações por pesticidas em crianças, adolescentes e jovens no município de Fortaleza. Ciênc Cuid Saude. 2009;8:48-55

13. Departamento de Informática do Sistema Único de Saúde. Indicadores de mortalidade: $C$. 9 Taxa de mortalidade específica por causas externas. 2014. Disponível em: http://tabnet. datasus.gov.br/cgi/deftohtm.exe?ibge/cnv/poppr.def. Acesso em: 15 nov. 2014.

14. Paranaguá. Profissionais do CAPS farão orientação sobre saúde mental amanhã. 2014. http://www.paranagua.pr.gov.br/noticias/noticia 6180.html. Acesso em: 8 ago. 2014.

15. Naghavi M, Shahraz S, Sepanlou SG, Dicker D, Naghavi P, Pourmalek F, et al. Health transition in Iran toward chronic diseases based on results of Global Burden of Disease 2010. Arch Iran Med. 2014;17:321-35.

16. Ougrin D, Banarsee R, Dunn-Toroosian V, Majeed A. Suicide survey in a London borough: primary care and public health perspectives. J Public Health (0xf). 2011;33:385-91.

17. Pompili M, Gonda X, Serafini G, Innamorati M, Sher L, Amore M, et al. Epidemiology of suicide in bipolar disorders: a systematic review of the literature. Bipolar Disord. 2013;15:45790.

18. Pinto LW, Pires TO, Silva CMFP, Assis SG. Suicide mortality temporal trends in people aged 60 years or more in the Brazilian states: 1980 to 2009. Cienc Saude Colet. 2012;17:1973-81.

19. International Association for Suicide Prevention. World suicide prevention day: suicide prevention across the globe: strengthening protective factors and instilling hope. 2012. Disponivel em: https://www.iasp.info/wspd/2012_wspd.php. Acesso em: 8 jan. 2015.

20. Minayo MCS, Cavalcante FG. Estudo compreensivo sobre suicídio de mulheres idosas de sete cidades brasileiras. Cad Saude Publica. 2013;29:2405-15.

21. Minayo MCS, Pinto LW, Assis SG, Cavalcante FG, Mangas RMNM. Tendência da mortalidade por suicídio na população brasileira e idosa, 1980-2006. Rev Saude Publica. 2012;46:300-9.

22. Lovisi GM, Santos SA, Legay L, Abelha L, Valencia E. Análise epidemiológica do suicídio no Brasil entre 1980 e 2006. Rev Bras Psiquiatr. 2009:32:86-93.

23. VärnikP. Suicide in the world. Int J Environ Res Public Health. 2012:9:760-71.

24. Brasil. Ministério da Saúde. Secretaria de Vigilância em Saúde. Departamento de Análise de Situação de Saúde. Saúde Brasil 2011: uma análise da situação de saúde e a vigilância da saúde da mulher. Brasilia, DF, 2012. 